\title{
Simulation and Research of the Nozzle with an Ultrasonic Resonator for Spraying Polymeric Materials
}

\author{
Serhiy HORIASHCHENKO*, Ievgeniia GOLINKA**, Algimantas BUBULIS**, \\ Vytautas JURENAS $* *$ \\ *Khmelnytsky National University, st Institutskaya st. 11, 29016, Khmelnitskiy, Ukraine, E-mail: gsl7@ukr.net \\ **Kaunas University of Technology, Studentu 56-338, LT 51424 Kaunas, Lithuania, E-mail: ievgeniia.golinka@ktu.edu \\ **Kaunas University of Technology, Studentu 56-338, LT 51424 Kaunas, Lithuania, E-mail: algimantas.bubulis@ktu.lt, \\ **Kaunas University of Technology, Studentu 56-338, LT 51424 Kaunas, Lithuania, E-mail: vytautas.jurenas@ktu.lt
}

cross $^{\text {ref }}$ http://dx.doi.org/10.5755/j01.mech.24.1.20215

\section{Introduction}

Spraying a variety of liquids and spray coating is the basis for a significant number of manufacturing processes in industries related. First of all, to the high-tech sectors of the economy. These include sprays, intended to work in the plant spectral analysis, spraying photoresist, polishing liquids in opto-electronic instrument, the coating in the manufacture of medical devices and implants, spraying fluids in the production of functional nanomaterials and more $[1,2$, 3].

For the application of polymeric materials on the surface of various tissues by gas-dynamic use special devices for their spraying. However, the quality of the coating is not permanent. This is due to several factors: the dispersion droplets uneven spraying flame front, the external environment. To improve the characteristics of each need an appropriate set of measures. On improving the dispersion of droplets affects the parameters of injector nozzle. Optimization of these parameters have an urgent task. To solve these problems it is necessary to develop effective methods of spraying liquids $[4,5]$.

One interesting studies revealed that the spraying time and liquid polymer substance having acoustic vibrations in the flow [6]. Consequently, considering this study, it is necessary to take into account that not only the acoustic field generated by the piezoceramics gives results, but also the natural oscillations of the nozzle. Fortunately, the natural oscillation of the system is not so great as to prevent the splitting of the drop.

\section{Coherence and transfer functions approach}

Among the factors that determine the operating parameters of the injector process has important working pressure drop and polymer properties. The main parameters of the nozzle can usefully be measured by a simple model. Nozzle design must provide a given fineness liquid spray at minimum pressure. This is possible when the magnitude of the resistance movement of the nozzle is minimal fluid in the channels. Therefore, the channels must be kept short.

As is well known, the use of ultrasonic waves provides a uniform distribution of droplet diameters. Furthermore, they become much smaller. This leads to an increase in the quality of the applied layer. However, only the use of ultrasonic waves to disperse the polymer is insufficient. It is proposed to combine a classical spray nozzle for the gas- dynamic method of polymer compositions with an ultrasonic resonator.

In place of the beam of ultrasonic waves, when spraying in the fountain on the surface of the jet excited capillary waves. The reason for the excitation of capillary waves are pressure surges due to the collapse of cavitation bubbles. For ultrasonic fountain necessary frequency megahertz range. In the upper part of the fountain spraying liquid, thereby forming a stable monodisperse aerosol with an average droplet size of about 2 - $4 \mathrm{~mm}$. [7, 8].

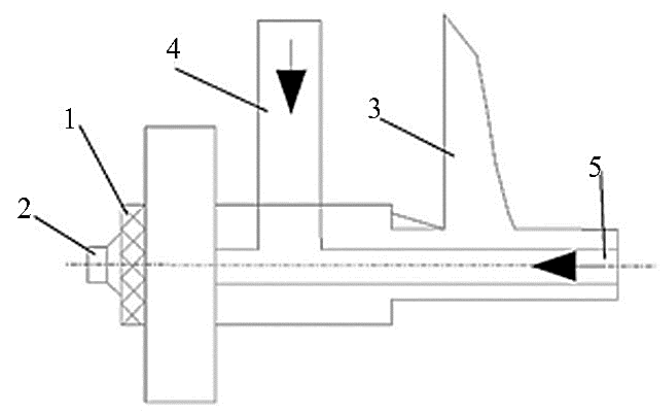

Fig. 1 Schematic view of the device

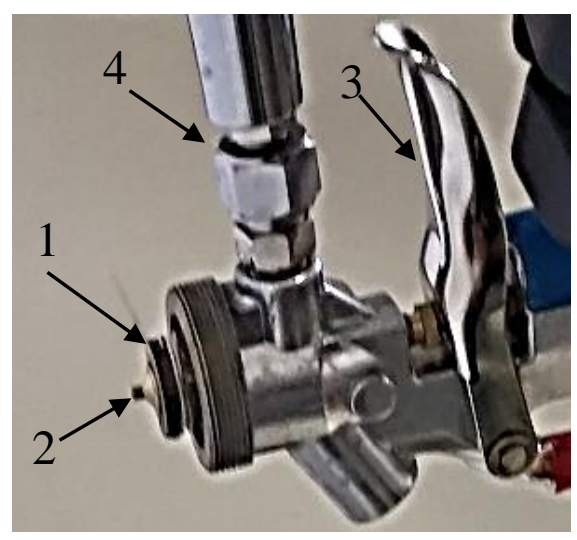

Fig. 2 The actual device image

When using ultrasonic frequencies over $103 \mathrm{kHz}$ may be changes in the structure of materials, electronic excitation, magnetic and electro-acoustic effect. This frequency range is most often used in acoustic, physical and chemical methods of analysis for the study of the nature and magnitude of intermolecular interactions, acoustic parameters of the environment studies, conformational transformations. 
Exterior spray apparatus shown in Fig. 1, where 1 - piezo-ceramic disc, 2 - spray nozzle, 3 - control knob, 4 the tube for supplying the polymer, 5 - tube for compressed air. The actual image of the device is presente in Fig. 2. The ultrasonic generator and the nozzle located on an atomizer in Fig. 3.
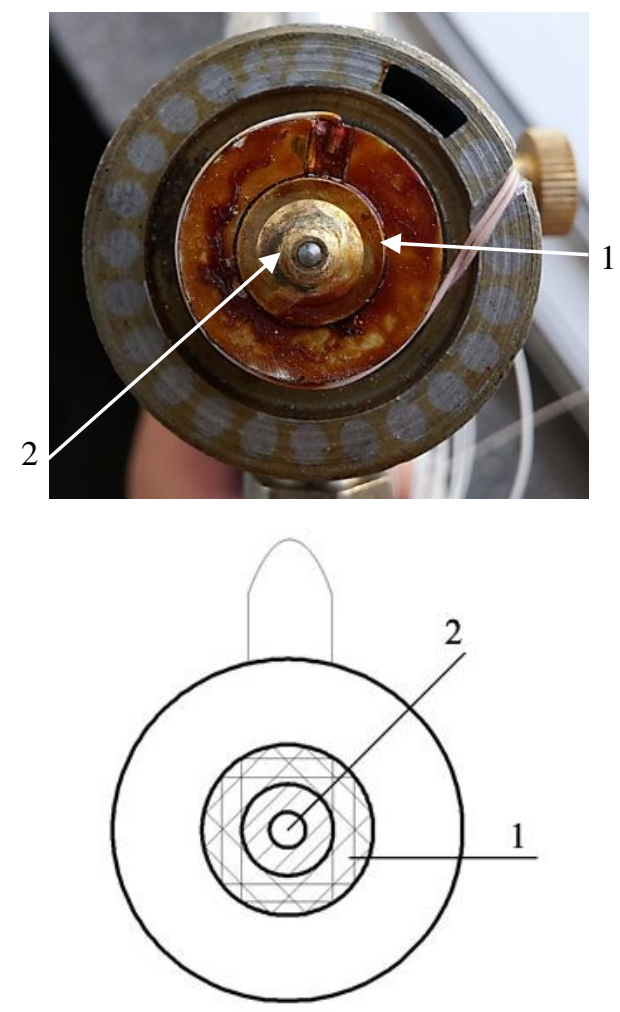

Fig. 3 Mounting the piezoelectric element around the sprayer nozzle: 1 - piezo-ceramic ring; 2 - spray nozzle

The model of the sprayer gun was made and simulate a stream of particles. For a first look at the flow of the spray (Fig.4), which shows 1- application surface, 2 ultrasonic sprayer gun.
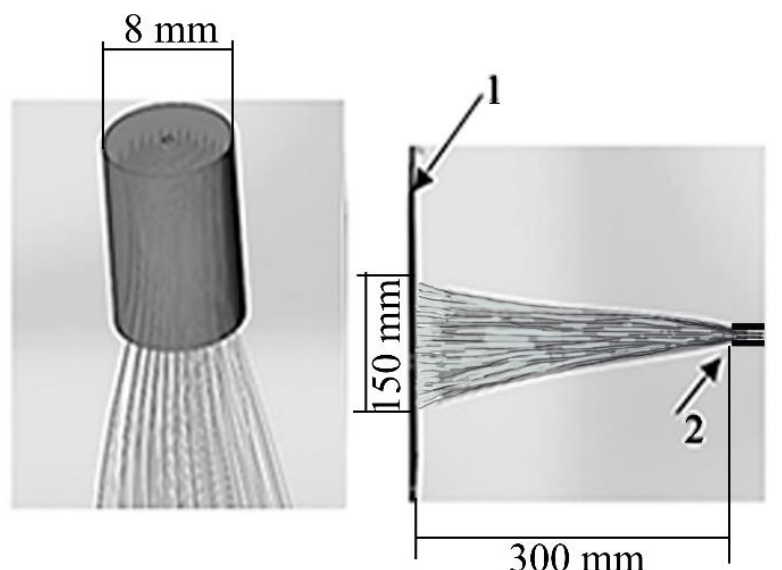

Fig. 4 Model of the nozzle and flow that is spray on the surface

Create a model of a nozzle with an ultrasonic resonator. The result is shown in Fig. 4. The distance between points 1 and 2 is $30 \mathrm{~cm}$. This distance is optimal for increasing the spray area and the pour point of the polymer drop when cooling in the air stream. At this distance, the spray area is $15-\mathrm{cm}$. Modeled distribution of vibrational waves from the ultrasonic resonator, which will be distribute in the spray stream. The frequency of oscillation of the polymer flow can be from 20 to $150 \mathrm{kHz}$.

The simulation results showed that there is a difference on the movement of particles in the middle and on the edge of the spray cone. In addition, the use of the ultrasonic resonator smoothed the distribution of the front of the spray stream.
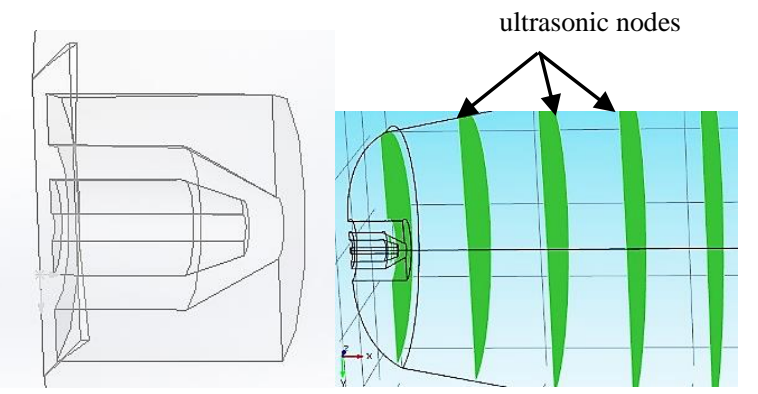

Fig. 5 Model of the nozzle with an ultrasonic resonator and simulation of vibrations

\section{Measurement system and data collection}

The investigation of the vibration of the ultrasonic spray nozzle and the cavity. Polytec 3D scanner equipment is used to accurately detect movements (Fig. 6: 1 -scanners head, 2 - object of study, anti-vibration table). Place the point of laser measuring devices on the spray gun so that only they covered the nose portion of the nozzle. Create a grid on the ultrasonic resonator (Fig. 7).

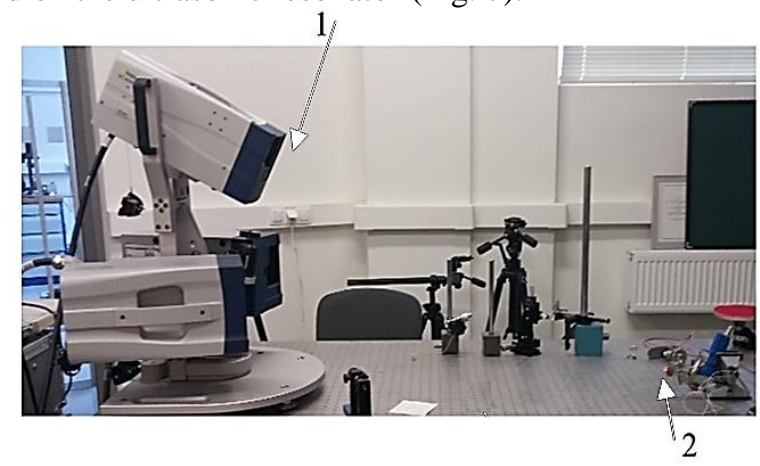

Fig. 6 Equipment to the precise determination of the displacements Polytec 3D scanner
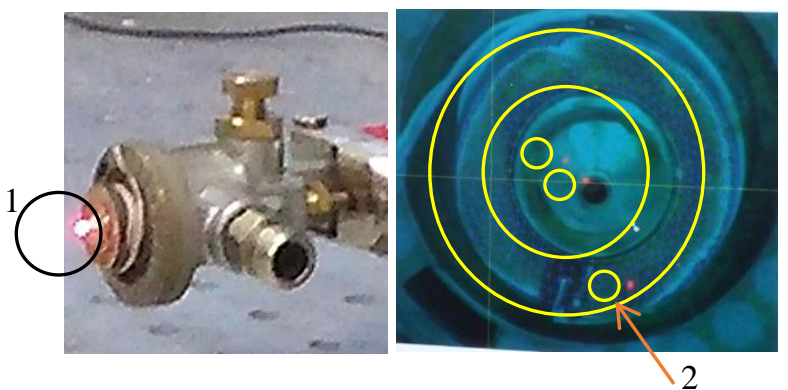

Fig. 7 Location of measurement points and the establishment of the grid scanning ultrasonic resonator: 1 position of the laser scanner, 2 - zone, which scan on the displacement

The experiment was conducted with several replays. As a result, graphs were prepared by changing the 
oscillation amplitude at the resonator frequency. Were also measured natural frequency of oscillation of the nozzle bases and nasal part. Nozzle vibration frequency measurement was conducted with included an ultrasonic resonator. The result is present on Fig. 8.

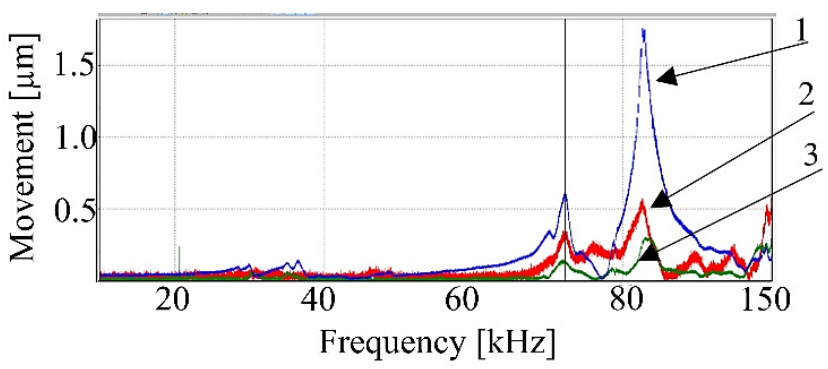

Fig. 8 Movements of the nozzle fixed during operation of the piezoceramic radiator, where: $1-\mathrm{X}$-axis; $2-\mathrm{Y}$ axis; 3 - Z-axis.

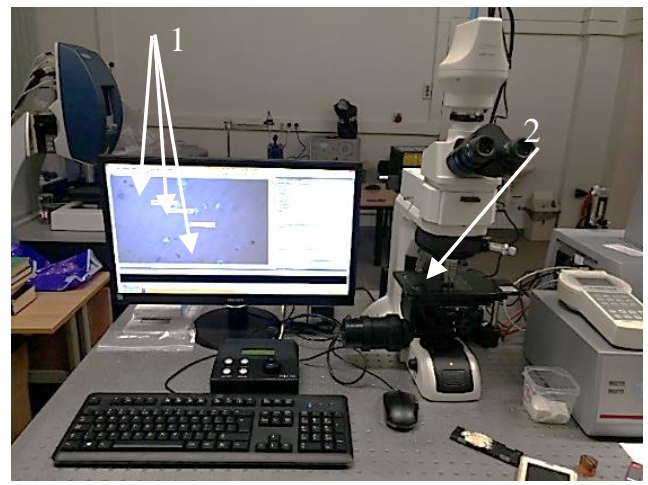

Fig. 9 Analysis of fused polymers in structure of tissue: 1 - the image on the cloth sprayed polymer droplets; 2 - research object when viewed under a microscope

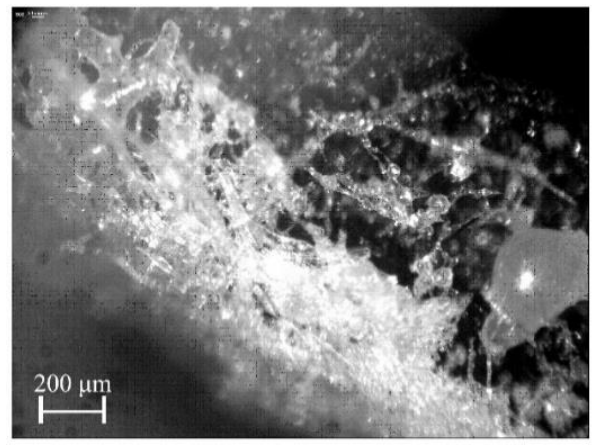

a

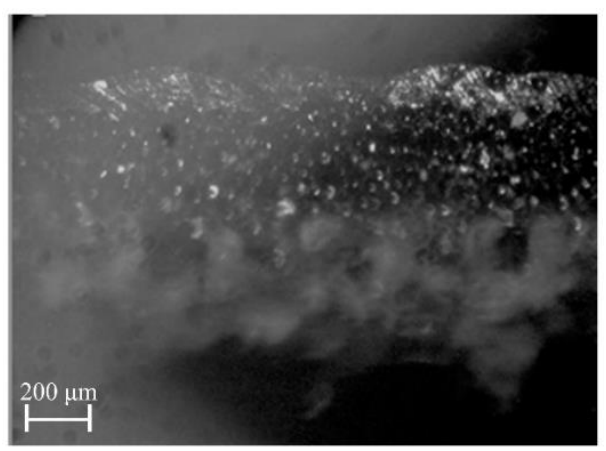

b

Fig. 10 Investigation of the surface of a polymer coated fabric. a) without the use of ultrasonic radiation; b) using ultrasonic radiation
It was made by spray nozzle such polymers with a resonator. The results were obtained using a microscope $\mathrm{Ni}$ kon microscope Eclipse LV100. Exterior view of the microscope and the internal distribution of the polymer in the tissue structure are show in Fig. 9. It can be seen spreading the polymer inside, which is typical for finely dispersed fluid.

As can be seen from the obtained images, when using an ultrasonic radiator (Fig. 10, b), polymer droplets are broken into small dots and there are no glue agglomerations. Undoubtedly, this fact improves the quality of the applied coating and allows reducing the polymer consumption, as well as the final weight of the product.

\section{Conclusion}

Studies have fully confirmed the hypothesis about the possibility of obtaining a more uniform flow of the spray when applying ultrasonic resonator. It was carried out computer simulations of the spray nozzle fluid flow and oscillation frequency. The boundaries of the ultrasonic excitation 20-100 kHz. Experimental study of the nozzle oscillation and generator both separately and together. A resonant frequency in the $72.38 \mathrm{kHz}$ was obtained. An increase in the resonant vibrations 5 times, which led to a better grinding of the spray droplets of liquid polymer. This result can be seen on the microscope. The thickness of the polymeric fibers less than 0.25 microns. The data obtained from Politec 3D indicate that the greatest deformation occurs along the $\mathrm{X}$ axis. This characteristic indicates the presence of a standing ultrasonic wave in the direction of atomization of the polymers. Ultrasound and radiation pressure contribute to a better crushing of polymer droplets, which was noted by us. Spreading polymer liquid occurred in the whole structure of the fabric material. The results can be used for the design of new equipment with improved spray stream polymers.

\section{References}

1. Ashish Kumar Sen. 2001. Coated textiles:Principles and Applications. Technomic Publishing Company, Inc -Kanpus, 2001, 228.

2. Ferrazza, J.; Bartell, W.; Schick, R. 1992. Spraying Systems Co. Spray nozzle drop size: How to evaluate measurement techniques and interpret data and reporting procedures. Bulletin 336.

3. Maetsky, A.V. 2012. Overview of computational methods of fuel atomization quality study diesel injector and analysis of factors affecting the quality of atomization, Young scientist. - 2012. - Vol (3), 61-70.

4. Walter, F. 2002. Coated and laminated textiles, Published by Woodhead Publishing Limited in association with The Textile Institute, Woodhead Publishing Ltd Abington Hall, AbingtonWoodhead Publishing ISBN 1855735768, 2002, 422.

5. Horyashchenko, S; Paraska, G; Petegerych, S; 2012. Modeling and research of polymer coating on clothing materials, Innovation in textile materials\&protective clothing. Monograph, Warsaw, 2012, 151-159.

6. Arthurs, D.; Samir, Z. 2011. The planar jet-plate oscillator, Journal of Fluids and Structures, Vol (27), 105120.

7. E1620-97: Standard Terminology relating to liquid particle statistics. Book of ASTM Standards, General Methods and Instrumentation, Volume 14.02: 810-812. 
8. Liubchyk, V.; Kylimnik, A.; Horyashchenko, S. 2013. Application of the Multi-frequency Phase Method of Ranging to Many Objects for Construction of Ground Penetrating Radar, International Radar Symposium (IRS-2013), Dresden, Germany, 19 - 21 May , 2013. 835-840.
S. Horiashchenko, I. Golinka, A. Bubulis, V. Jurenas

SIMULATION AND RESEARCH OF THE NOZZLE WITH AN ULTRASONIC RESONATOR FOR SPRAYING POLYMERIC MATERIALS

S u m m a r y

In this paper, a method for the aerosol application of polymers to fabric surfaces is discussed. A lot of thermoplastic polymer materials during application have the property of hardening and the formation of glue lumps. This leads to a decrease in the quality of the coating applied. In view of the fact that aerosol coating is undoubtedly advantageous, it required improvement. Modeling of polymer flow through the nozzle was perform to determine the optimal application distance. The experimental part consisted of applying a solution of a polyvinyl chloride polymer to a fabric using an ultrasonic radiator on a diffuser. Just for comparison, samples without the connection of the radiator were perform. Politec 3D scanner equipment used to determine deformation changes. This study has a weight for the further development and improvement of coating devices.

Keywords: nozzle, spraying, frequency, ultrasound.

Received January 10, 2017

Accepted February 15, 2018 